\title{
Influência da concentração de lodo sobre o consumo de energia para aeração em sistemas de lodo ativado
}

\author{
Influence of the sludge concentration on the energy \\ consumption for aeration in activated sludge systems \\ Silvânia Lucas dos Santos', Vanessa Gomes Miná2, \\ Sebastian Yuri Cavalcanti Catunda ${ }^{3}$, Adrianus Cornelius Van Haandel ${ }^{2,4}$
}

口-

\begin{abstract}
RESUMO
Este trabalho teve como objetivo avaliar quantitativamente a influência da concentração de lodo sobre os fatores que afetam a capacidade de aeração em sistemas de lodo ativado. A investigação experimental foi desenvolvida aplicando a técnica da respirometria, que possibilitou a determinação da taxa de consumo de oxigênio e das variáveis que afetam a transferência de oxigênio. Os resultados experimentais indicam que a concentração de lodo afeta de maneira significativa a capacidade de aeração em sistemas de lodo ativado e, consequentemente, aumenta consideravelmente o consumo de energia. A viabilidade econômica de sistemas que permitem aumento da concentração de lodo, como reatores biológicos com membranas e reatores biológicos com leito móvel, é questionável quando se comparam os custos de implementação com os benefícios resultantes.
\end{abstract}

Palavras-chave: concentração de lodo; respirometria; capacidade de aeração.

\begin{abstract}
The objective of this research was to evaluate quantitatively the influence of sludge concentration on the factors affecting the aeration capacity in activated sludge systems. The experimental investigation involved the application of respirometry, by which the oxygen consumption rate was determined and allowed evaluation of the effect of concentration on the variables that affect oxygen transfer The experimental results indicated that the sludge concentration has a strong effect on aeration capacity and consequently on the energy consumption. The economic feasibility of systems that allow operation at high concentration, like membrane bioreactor and moving bed biofilm reactor, is questionable when the costs of implementation are compared to the resulting benefits.
\end{abstract}

Keywords: sludge concentration; respirometry; aeration capacity.

\section{INTRODUÇÃO}

Os reatores biológicos com membranas (MBR) e os reatores biológicos com leito móvel (MBBR) são novas variantes do sistema de lodo ativado; esses reatores são usados para operar com concentrações de lodo bem mais elevadas que as verificadas nos sistemas convencionais (DEZOTTI; JÚNIOR SANT'ANNA; BASSIN, 2011). Isso possibilita a operação de sistemas com volumes menores, o que pode reduzir os custos de construção, além de ser importante pela limitação do espaço para a implantação dos sistemas. No entanto, a consequência da diminuição do volume é o aumento da concentração do lodo no sistema, que poderá afetar a capacidade de transferência de oxigênio. Os fatores que podem ser afetados pelo aumento da concentração de lodo são: 1. a constante de transferência de oxigênio $\left(\mathrm{K}_{\mathrm{la}}\right)$;
2. a concentração do oxigênio de saturação $\left(\mathrm{OD}_{\mathrm{s}}\right)$; e

3. a concentração do oxigênio mínima ou crítica $\left(\mathrm{OD}_{\mathrm{cr}}\right)$ necessária para a operação adequada do sistema de lodo ativado (VAN HAANDEL \& CATUNDA, 2013).

Uma alta concentração de lodo tenderá a reduzir a capacidade de aeração, de modo que, para atender a uma determinada concentração de oxigênio dissolvido no sistema, os gastos energéticos bem como os custos operacionais aumentarão (MEDEIROS et al.,2008).

Sob condições operacionais e cargas constantes, a massa de lodo em sistemas de lodo ativado e a demanda diária de oxigênio são constantes (VAN HAANDEL \& MARAIS, 1999). No entanto, a eficiência da aeração expressa, como a massa de oxigênio transferido por unidade

'Doutora em Engenharia Ambiental na Universidade Estadual da Paraíba - Campina Grande (PB), Brasil.

${ }^{2}$ Mestre em Ciência e Tecnologia Ambiental pela Universidade Estadual da Paraíba - Campina Grande (PB), Brasil.

${ }^{3}$ Doutor em Engenharia Elétrica. Professor da Universidade Federal do Rio Grande do Norte - Natal (RN), Brasil.

${ }^{4}$ Doutor em Engenharia Civil. Professor da Universidade Federal de Campina Grande - Campina Grande (PB), Brasil.

Endereço para correspondência: Silvânia Lucas dos Santos - Rua José Evaristo Barbosa, 323 - 58410-380 - Catolé - Campina Grande (PB), Brasil - E-mail: silvania_sls@hotmail.com Recebido: 29/07/13 - Aceito: 17/03/17 - Reg. ABES: 121436 
de energia elétrica necessária para efetuar a aeração, não é constante e tende a variar com a concentração de oxigênio dissolvido (OD). A taxa de transferência de oxigênio pode ser expressa pela lei de Fick, a qual afirma que a taxa de aeração é proporcional ao déficit de oxigênio, a diferença entre a concentração de saturação e a concentração de oxigênio na fase líquida $\left(\mathrm{OD}_{1}\right)$ em contato com o aerador (METCALF \& EDDY, 2003). Portanto, essa taxa pode ser escrita como na Equação 1:

$\mathrm{r}_{\mathrm{ae}}=\left(\mathrm{dDO}_{\mathrm{l}} / \mathrm{dt}\right)_{\mathrm{a}}=\mathrm{K}_{\mathrm{la}}\left(\mathrm{OD}_{\mathrm{s}}-\mathrm{OD}_{\mathrm{l}}\right)$

Em que:

$\mathrm{r}_{\mathrm{ae}}=$ taxa de aeração;

$\mathrm{K}_{\mathrm{la}}=$ constante de transferência de oxigênio $\left(\mathrm{h}^{-1}\right)$;

$\mathrm{OD}_{\mathrm{s}}=$ concentração de oxigênio dissolvido de saturação $\left(\mathrm{mg} \cdot \mathrm{L}^{-1} \cdot \mathrm{h}^{-1}\right)$;

$\mathrm{OD}_{1}=$ concentração de OD no licor misto $\left(\mathrm{mg} \cdot \mathrm{L}^{-1}\right)$.

Normalmente, o efeito da absorção de oxigênio atmosférico é insignificante, mas em caso de utilização de reatores muito pequenos com área superficial relativamente alta, a taxa de variação de OD pode ser afetada pelo efeito da absorção. Nesse caso, a taxa de aeração se dá pela combinação do efeito da aeração mecânica e da absorção de oxigênio atmosférico; nessas circunstâncias, a Equação 1 pode ser reescrita como na Equação 1a:

$\mathrm{r}_{\mathrm{a}}=\mathrm{r}_{\mathrm{ae}}+\mathrm{r}_{\mathrm{abs}}=\mathrm{K}_{\mathrm{la}}\left(\mathrm{OD}_{\mathrm{s}}-\mathrm{OD}_{\mathrm{l}}\right)+\mathrm{K}_{\mathrm{abs}}\left(\mathrm{OD}_{\mathrm{s}}-\mathrm{OD}_{\mathrm{l}}\right)=\mathrm{K}_{\mathrm{la}}^{\prime}\left(\mathrm{OD}_{\mathrm{s}}-\mathrm{OD}_{\mathrm{l}}\right)$

Em que:

$\mathrm{K}_{\mathrm{abs}}=$ constante de absorção de oxigênio atmosférico $\left(\mathrm{h}^{-1}\right)$;

$\mathrm{K}_{\mathrm{la}}^{\prime}=$ constante de transferência de oxigênio - efeito aeração mecânica + absorção $\left(h^{-1}\right)$.

A taxa de transferência máxima de oxigênio é determinada pela concentração mínima necessária para manter o OD suficiente para o desempenho adequado do sistema de lodo ativado, como expressa a Equação 2. A concentração de oxigênio no licor misto pode influenciar uma série de fatores, tais como a eficiência de remoção do substrato, a sedimentabilidade do lodo e a atividade das bactérias autotróficas e heterotróficas presentes, que, por sua vez, determinam a capacidade de tratamento do sistema de lodo ativado. Portanto:

$\mathrm{CO}_{\mathrm{m}}=\mathrm{K}_{\mathrm{la}}\left(\mathrm{OD}_{\mathrm{s}}-\mathrm{OD}_{\mathrm{c}}\right)$

Em que:

$\mathrm{CO}_{\mathrm{m}}=$ capacidade de aeração ou taxa máxima de transferência de oxigênio por volume de reator $\left(\mathrm{g} \cdot \mathrm{L}^{-1} \cdot \mathrm{h}^{-1}\right)$;

$\mathrm{OD}_{\mathrm{c}}=$ concentração crítica de oxigênio que deve ser mantida na zona de aeração $\left(\mathrm{mg} \cdot \mathrm{L}^{-1}\right)$.
Recentemente, novas variantes do processo de lodo ativado, particularmente o MBR e o MBBR, vêm sendo aplicadas como alternativas que podem reduzir o volume necessário do reator (VEOLIA, 2009; ODEGAARD; GISVOLD; STRICKLAND, 2000; VAN HAANDEL \& VAN DER LUBBE, 2012). Isso é possível porque essas variantes podem ser operadas a uma concentração de lodo na faixa de 10 a 15 g.L.valores mais elevados que os encontrados em sistemas de lodo ativado convencional, nos quais a concentração no tanque de aeração é tipicamente entre 3 e 6 g.L.-1 . No entanto, a maior concentração de lodo afetará as propriedades reológicas do licor misto e, consequentemente, a transferência de oxigênio da atmosfera para o licor misto. Em princípio, todos os três fatores que influenciam a capacidade de aeração de um aerador, conforme a Equação 2, podem ser afetados pelo aumento da concentração de lodo.

Em sistemas de lodo ativado, a transferência de oxigênio para a fase líquida deve contrabalançar o consumo de oxigênio pela massa bacteriana. Nesta pesquisa, a técnica da respirometria foi empregada para a determinação da taxa de consumo de oxigênio (TCO). Assim, foi possível avaliar a influência da concentração de lodo sobre a capacidade de oxigenação e os gastos com energia necessários para atender à aeração necessária para os processos de tratamento do sistema de lodo ativado.

\section{METODOLOGIA}

O lodo utilizado para execução da investigação experimental foi proveniente de um sistema de lodo ativado em escala de laboratório, sendo alimentado diariamente com esgoto doméstico, e operado com idade de lodo de 5 dias, tempo insuficiente para a nitrificação. O sistema de geração de lodo utilizado nesta pesquisa operou no regime de bateladas sequenciais. Foi construído em acrílico, $\operatorname{com} 22 \mathrm{~cm}$ de diâmetro, $50 \mathrm{~cm}$ de altura de forma cilíndrica e volume útil de $14 \mathrm{~L}$.

O lodo produzido foi utilizado para avaliar a influência da concentração do lodo sobre a capacidade de oxigenação (CO). Do licor misto de descarte do sistema de lodo ativado era retirado por sedimentação aproximadamente $1 \mathrm{~L}$ de lodo concentrado (cerca de 15 g.L $\mathrm{L}^{-1}$ de sólidos totais), que era submetido à aeração intermitente. A aeração era produzida por uma pequena bomba de ar que insuflava bolhas dentro do reator por meio da utilização de pedras porosas; o reator utilizado para os testes laboratoriais foi um béquer com capacidade de $2 \mathrm{~L}$. A pedra porosa foi colada na parte inferior do reator, de modo que sua posição e as condições de aeração se mantiveram sempre constantes.

Para a realização dos testes respirométricos, foi utilizado o respirômetro Beluga, que tem a função de armazenar em planilhas

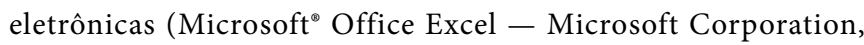
Redmond, Washington, Estados Unidos) dados de TCO, OD e temperatura em função do tempo. Por meio desses dados gerados 
pelo respirômetro é possível determinar as constantes: $\mathrm{K}_{\mathrm{la}}, \mathrm{K}_{\mathrm{abs}}$, CO e oxigênio crítico. O respirômetro determina a TCO por meio do método clássico, conforme descrito por Catunda et al. (1996). Nesse método, o respirômetro faz com que a concentração de OD oscile entre um valor máximo e um mínimo, ambos preestabelecidos. Quando a concentração de OD está abaixo do máximo, a aeração está ligada e a concentração aumentará até OD chegar ao ponto de OD máximo. Nesse momento, a aeração é desligada e, devido ao consumo bacteriano, a concentração de OD diminui até o valor mínimo, enquanto o reator continua sendo agitado mecanicamente, para garantir uma concentração uniforme de lodo e a disponibilidade de substrato para toda a massa microbiana. Os dados da TCO eram calculados, por regressão linear, pelo respirômetro, como expressa a Equação 3:

$\mathrm{TCO}=\left(\mathrm{OD}_{\max }-\mathrm{OD}_{\min }\right) /(\Delta \mathrm{t})$

Em que:

TCO = taxa de consumo de oxigênio $\left(\mathrm{mg} \cdot \mathrm{L}^{-1} \cdot \mathrm{h}^{-1}\right)$;

$\mathrm{OD}_{\text {máx }}=$ valor de oxigênio dissolvido de referência superior $\left(\mathrm{mg} . \mathrm{L}^{-1}\right)$; $\mathrm{OD}_{\min }=$ valor de oxigênio dissolvido de referência inferior $\left(\mathrm{mg} . \mathrm{L}^{-1}\right)$; $\Delta t$ = intervalo de tempo entre $\mathrm{OD}_{\text {máx }}$ e o $\mathrm{OD}_{\text {min }}\left(\mathrm{h}^{-1}\right)$.

\section{Determinação da constante de transferência de oxigênio}

Neste trabalho, é apresentado um método para determinar a influência da concentração de lodo sobre a taxa de transferência de oxigênio usando a respirometria para a determinação das variáveis $\mathrm{K}_{\mathrm{la}}, \mathrm{OD}_{\mathrm{s}} \mathrm{e}$ $\mathrm{OD}_{\text {cr }}$ testadas para diferentes concentrações de lodo.

A concentração de $\mathrm{OD}_{\text {max }}$ deve ser escolhida de maneira que, ao ser iniciada a aeração, o seu valor não seja alcançado em um período de tempo muito curto. A referência inferior não deve ser menor que a concentração mínima de OD, quando ela se torna um fator limitante no consumo e, consequentemente, reduz a TCO. Por essa razão, geralmente o limite inferior de OD no tanque de aeração é de $1 \mathrm{mg}$. $\mathrm{L}^{-1}$. Durante os períodos com aeração, o aumento da concentração de OD pode ser expresso como na Equação 4:

$\mathrm{dOD}_{\mathrm{l}} / \mathrm{dt}=\mathrm{r}_{\mathrm{ae}}-\mathrm{TCO}=\mathrm{K}_{\mathrm{la}}\left(\mathrm{OD}_{\mathrm{s}}-\mathrm{OD}_{\mathrm{l}}\right)-\mathrm{TCO}$

Em que:

$\mathrm{dOD}_{\mathrm{l}} / \mathrm{dt}=$ taxa de variação dos valores da concentração de OD.

A Equação 4 pode ser resolvida se a TCO não se alterar significativamente durante o período de aeração, como expressa na Equação 5:

$\mathrm{OD}_{\mathrm{l}}=\left(\mathrm{OD}_{\mathrm{s}}-\mathrm{TCO} / \mathrm{K}_{\mathrm{la}}\right) *\left(1-\exp \left(-\mathrm{K}_{\mathrm{la}} \mathrm{t}\right)\right)+\mathrm{OD}_{\mathrm{o}} \exp \left(-\mathrm{K}_{\mathrm{la}} \mathrm{t}\right)$
Em que:

$\mathrm{K}_{\mathrm{la}}=$ constante de transferência de oxigênio $\left(\mathrm{h}^{-1}\right)$;

$\mathrm{T}=$ tempo $\left(\mathrm{h}^{-1}\right)$;

$\mathrm{OD}_{\mathrm{o}}=$ concentração de OD no início do período de aeração $\left(\mathrm{mg} \cdot \mathrm{L}^{-1}\right)$.

A Equação 5 pode ser usada para determinar experimentalmente os valores de $\mathrm{K}_{\mathrm{la}}$ e OD para qualquer concentração de lodo, se a concentração de OD no licor misto for medida em função do tempo durante o período de aeração com um valor constante da TCO. Os valores experimentais de $\mathrm{OD}_{1}$ obtidos podem ser simulados, traçando curvas da Equação 5 para $\mathrm{OD}_{1}$ em função do tempo durante o período com aeração. Os valores de $\mathrm{K}_{\mathrm{la}}$ e $\mathrm{OD}_{\mathrm{s}}$ escolhidos serão aqueles que resultam na correlação mais próxima entre as curvas teóricas e os dados experimentais de $\mathrm{OD}_{1}$, obtidos pelo respirômetro. A alternativa mais prática é utilizar a ferramenta solver do Excel para o computador encontrar os valores das constantes $\mathrm{K}_{\mathrm{la}}$ e $\mathrm{OD}_{\mathrm{s}}$ de melhor correlação (sub-rotina "solver").

Como exemplo, na Figura 1 os valores medidos (círculos pequenos) do $\mathrm{OD}_{1}$ são representados em função do tempo de aeração. No mesmo gráfico, as curvas teóricas foram produzidas para diferentes valores de $\mathrm{K}_{\mathrm{la}}$ (à esquerda) e $\mathrm{OD}_{\mathrm{s}}$ (à direita). Observa-se que há uma correlação quase perfeita para valores de $\mathrm{K}_{\mathrm{la}}=1,6 \mathrm{~min}^{-1} \mathrm{e} \mathrm{OD}_{\mathrm{s}}=7,5 \mathrm{mg}$. $\mathrm{L}^{-1}$. Pode-se notar que no período inicial a simulação é imperfeita, quando a taxa de aeração é muito maior do que os valores de TCO e a concentração de $\mathrm{OD}$ aumenta rapidamente. Isso pode ser atribuído à relaxação do eletrodo de OD (que ocorre quando a concentração de OD muda rapidamente, a concentração medida de OD “corre atrás” da concentração verdadeira). Quando o aumento da concentração de OD e, portanto, a taxa de aeração se reduzem (Equação 1), a taxa de variação da concentração de OD diminui e a correlação entre os valores experimentais e teóricos se torna perfeita.

O terceiro fator que influencia a capacidade de aeração é a concentração de OD mínima que deve ser aplicada para evitar que o valor se torne um fator limitante para os processos biológicos. Na investigação experimental, observou-se que quando a aeração era interrompida, a relação OD/tempo permanecia linear até que a concentração de OD fosse quase zero, a menos que houvesse uma TCO muito elevada. Portanto, o valor da concentração mínima de OD era baixo para as bactérias heterotróficas.

No entanto, deve-se considerar que nos reatores em escala real sempre há uma estratificação da concentração de OD, com um máximo no ponto de introdução de ar e um mínimo à maior distância dos aeradores. A magnitude da estratificação depende do tipo do aerador e da configuração do reator, bem como da TCO e da intensidade de mistura. Para que toda a massa bacteriana tenha um valor acima da concentração mínima de OD necessária para que o oxigênio não seja fator limitante, deve-se considerar que, na prática, há uma concentração crítica 
de OD na zona da aeração, que é a soma da concentração mínima e da magnitude da estratificação.

\section{RESULTADOS E DISCUSSÃO}

Para estabelecer a influência da concentração de lodo sobre a constante de aeração, foram realizados ensaios respirométricos específicos que possibilitaram determinar $\mathrm{K}_{\mathrm{la}}$ para diferentes concentrações de lodo e avaliar sua influência sobre a capacidade de oxigenação. Como mostra o respirograma da Figura 2, na parte superior, indicado como gráfico A, observam-se vários ciclos do perfil de OD. Na parte inferior do respirograma, indicado como gráfico $\mathrm{B}$, observam-se os valores da TCO em função do tempo para as diferentes concentrações de lodo.

O respirograma da Figura 2 apresenta seis experimentos com diferentes concentrações de lodo (gráfico B). Nesse ensaio foi aplicado o método de determinação semicontínuo, intercalando períodos de aeração seguidos por períodos sem aeração, adotando-se com limites superior e inferior de OD 8 e $1 \mathrm{mgO}_{2} \cdot \mathrm{L}^{-1}$, respectivamente. Observa-se que, à medida que a concentração de lodo no reator diminui, a TCO também diminui. Em contrapartida, a concentração de oxigênio aumenta, aproximando-se de um valor constante, quando a TCO se iguala à taxa de aeração. A Equação 5 foi utilizada para determinar os melhores valores de $\mathrm{K}_{\mathrm{la}}$ e $\mathrm{OD}_{\mathrm{s}}$ para a simulação dos dados de $\mathrm{OD}_{1}$ obtidos durante os períodos de aeração.

$\mathrm{O}$ teste era realizado com $1 \mathrm{~L}$ de lodo concentrado. A cada registro de 3 pontos da TCO, realizava-se uma diluição de $25 \%$ da amostra original com o efluente do próprio sistema gerador de lodo, ocasionando uma redução na concentração de lodo. Um novo teste com uma amostra de $1 \mathrm{~L}$ menos concentrado era então iniciado: submetia-se novamente a amostra à aeração intermitente e novos valores de $\mathrm{K}_{\mathrm{la}}$ e $\mathrm{OD}_{\mathrm{s}}$

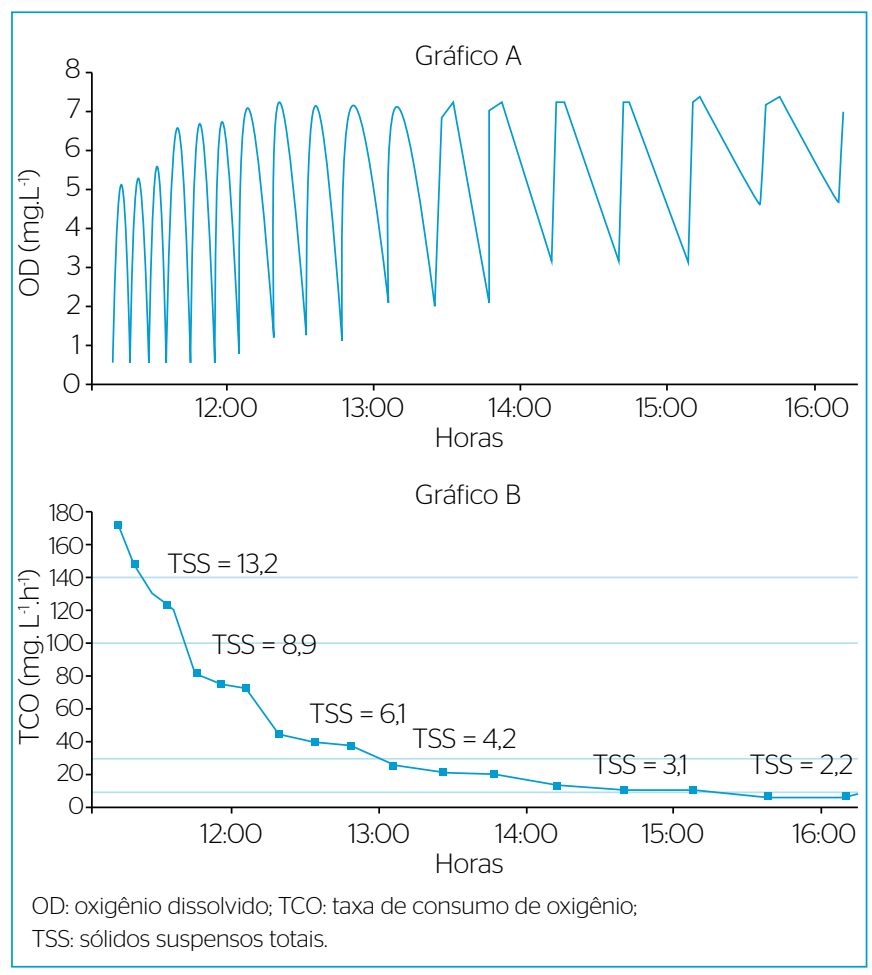

Figura 2 - Respirograma gerado pelo software do respirômetro com concentração de oxigênio dissolvido (A) e taxa de consumo de oxigênio (B) em função do tempo de aeração para diferentes concentrações de lodo.
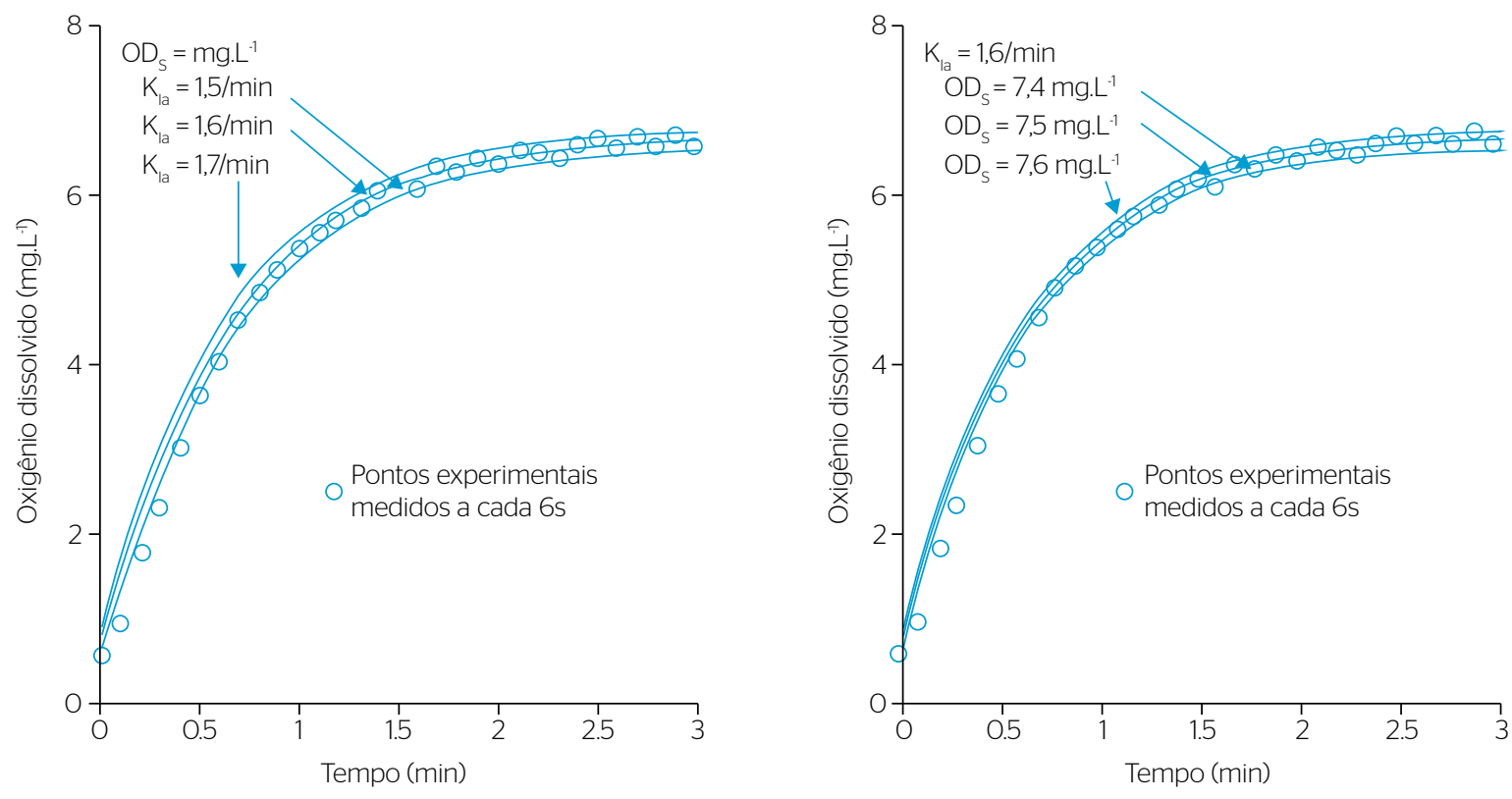

Figura 1 - Simulação dos valores de oxigênio dissolvido em função do tempo de aeração, com taxa de consumo de oxigênio constante. 
eram calculados. Após três ciclos, a concentração de lodo era novamente reduzida por outra diluição com efluente.

Para todas as etapas dos ensaios (exceto a última), foram obtidos 3 pontos experimentais de TCO, resultando em um total de 17 dados para TCO. Diferentes valores de $\mathrm{K}_{\mathrm{la}}$ e $\mathrm{OD}_{\mathrm{s}}$ foram obtidos por simulação para todos os testes, como mostra a Tabela 1. Nessa tabela, além desses valores, são apresentados os dados da média e do coeficiente de variação $(\mathrm{CV})$ para cada concentração.

Percebe-se, na Tabela 1, que para cada concentração pesquisada o valor de $\mathrm{K}_{\mathrm{la}}$ em boa aproximação é constante, mas há uma clara relação entre o $\mathrm{K}_{\mathrm{la}}$ e a concentração de lodo: a constante de transferência diminui consideravelmente na medida em que a concentração de lodo aumenta. Em contraste, os valores de $\mathrm{OD}_{\mathrm{s}}$ na Tabela 1 são praticamente constantes e independentes da concentração de lodo. Nesse caso, a concentração de saturação é melhor representada pela média de todos os testes: 7,55 mg.L-1.

Uma análise detalhada da Figura 2 mostra que, para a menor concentração (sólidos suspensos totais - SST = 2,2 g. ${ }^{-1}$ ), o perfil de $\mathrm{OD}_{1}$ em função do tempo desvia claramente da reta que se supõe (gráfico B). Isso deve ser atribuído à absorção de oxigênio atmosférico (mais notável quando a TCO é baixa). Para avaliar o efeito da absorção,

Tabela 1 - Valores experimentais da taxa de consumo de oxigênio, valores calculados de $\mathrm{K}_{\mathrm{la}}, \mathrm{OD}_{\mathrm{s}}$ e capacidade de oxigenação em função da concentração de lodo. A média e o coeficiente de variação também estão indicados.

\begin{tabular}{|c|c|c|c|c|c|c|c|c|}
\hline Teste & $\begin{array}{l}\text { SST } \\
\left(g \cdot L^{-1}\right)\end{array}$ & $\begin{array}{c}\text { TCO } \\
\left(m g \cdot L^{-1} h^{-1}\right)\end{array}$ & $\begin{array}{l}K_{1 a} \\
\left(h^{-1}\right)\end{array}$ & $\mid \begin{array}{c}\mathrm{K}_{\mathrm{la}} \\
\text { (Média) }\end{array}$ & $\begin{array}{l}\mathrm{CV} \\
(\%)\end{array}$ & $\begin{array}{l}\mathrm{OD}_{\mathrm{s}} \\
\left(\mathrm{mg} \cdot \mathrm{L}^{-1}\right)\end{array}$ & \begin{tabular}{|}
$\mathrm{OD}_{\mathrm{s}}$ \\
(média)
\end{tabular} & $\begin{array}{l}\text { CV } \\
\text { (\%) }\end{array}$ \\
\hline 1 & \multirow{3}{*}{13,2} & 172,8 & 58,8 & \multirow{3}{*}{58,2} & \multirow{3}{*}{1,20} & 7,6 & \multirow{3}{*}{7,67} & \multirow{3}{*}{1,8} \\
\hline 2 & & 149,5 & 57,5 & & & 7,8 & & \\
\hline 3 & & 129,9 & 58,5 & & & 7,6 & & \\
\hline 4 & \multirow{3}{*}{8,9} & 82,2 & 82,6 & \multirow{3}{*}{82,8} & \multirow{3}{*}{0,24} & 7,8 & \multirow{3}{*}{7,70} & \multirow{3}{*}{1,5} \\
\hline 5 & & 75,1 & 83,0 & & & 7,6 & & \\
\hline 6 & & 72,4 & 82,8 & & & 7,7 & & \\
\hline 7 & \multirow{3}{*}{6,1} & 44,3 & 95,2 & \multirow{3}{*}{96,0} & \multirow{3}{*}{0,73} & 7,6 & \multirow{3}{*}{7,60} & \multirow{3}{*}{0,8} \\
\hline 8 & & 40,1 & 96,3 & & & 7,6 & & \\
\hline 9 & & 37,9 & 96,5 & & & 7,6 & & \\
\hline 10 & \multirow{3}{*}{4,2} & 25,1 & 103,9 & \multirow{3}{*}{103,8} & \multirow{3}{*}{0,14} & 7,4 & \multirow{3}{*}{7,43} & \multirow{3}{*}{1,6} \\
\hline 11 & & 21,2 & 103,8 & & & 7,6 & & \\
\hline 12 & & 20,0 & 103,6 & & & 7,3 & & \\
\hline 13 & \multirow{3}{*}{3,1} & 12,5 & 113,8 & \multirow{3}{*}{114,0} & \multirow{3}{*}{0,22} & 7,4 & \multirow{3}{*}{7,43} & \multirow{3}{*}{0,8} \\
\hline 14 & & 11,5 & 114,3 & & & 7,4 & & \\
\hline 15 & & 10,9 & 114,0 & & & 7,5 & & \\
\hline 16 & \multirow{2}{*}{2,2} & 7,2 & 131,0 & \multirow{2}{*}{130} & \multirow{2}{*}{0,54} & 7,5 & \multirow{2}{*}{7,55} & \multirow{2}{*}{0,9} \\
\hline 17 & & 7,5 & 130,0 & & & 7,6 & & \\
\hline
\end{tabular}

SST: sólidos suspensos totais; TCO: taxa de consumo de oxigênio; $\mathrm{K}_{\mathrm{l}: \text { : }}$ constante de transferência de oxigênio; $C V$ : coeficiente de variação; $\mathrm{OD}_{s}$ : concentração de oxigênio de saturação. realizou-se um teste com água desoxigenada em vez de lodo (gráfico A) e determinou-se que a absorção de fato ocorria e que o valor da constante de absorção era $\mathrm{K}_{\mathrm{abs}}=2,4 \mathrm{~h}^{-1}$. Como esse valor é muito pequeno em comparação aos valores da $\mathrm{K}_{\mathrm{la}}$ (na faixa de $100 \mathrm{~h}^{-1}$ [Tabela 1]), desconsiderou-se o efeito da absorção.

$\mathrm{Na}$ Tabela 2, é possível observar, em função da concentração de lodo, os valores médios de TCO, $\mathrm{K}_{\mathrm{la}}$ e $\mathrm{OD}_{\mathrm{s}}$. Para calcular a capacidade de aeração, é necessário, ainda, que se defina o valor da concentração crítica de oxigênio $\left(\mathrm{OD}_{\mathrm{cr}}\right)$. Foi realizada uma série de testes para estabelecer o valor da concentração crítica de OD em função da TCO. Os resultados estão resumidos na Figura 3, em que pode ser visto que o OD cai linearmente com o tempo até que a concentração mínima seja alcançada. Essa concentração mínima depende da TCO. De um grande número de perfis, como mostra a Figura 3, a seguinte

Tabela 2 - Valores experimentais de taxa de consumo de oxigênio, $K_{\mathrm{ta}^{\prime}}$ $\mathrm{OD}_{\mathrm{s}}$ e $\mathrm{OD}$ em função da concentração de lodo. Também é indicado o valor relativo do consumo de energia.

\begin{tabular}{c|c|c|c|c|c|c}
\hline 1 & 2 & 3 & 4 & 5 & 6 & 7 \\
\hline $\begin{array}{c}\mathrm{SST} \\
\left(\mathrm{g} \cdot \mathrm{L}^{-1}\right)\end{array}$ & $\begin{array}{c}\mathrm{TCO} \\
\left(\mathrm{mg} \cdot \mathrm{L}^{-1} \cdot \mathrm{h}^{-1}\right)\end{array}$ & $\begin{array}{c}\mathrm{K}_{\mathrm{la}} \\
\left(\mathrm{h}^{-1}\right)\end{array}$ & $\begin{array}{c}\mathrm{OD}_{\mathrm{s}} \\
\left(\mathrm{mg}^{-1}\right)\end{array}$ & $\begin{array}{c}\mathrm{OD}_{\mathrm{c}} \\
\left(\mathrm{mg}^{-1}\right)\end{array}$ & $\begin{array}{c}\mathrm{CO} \\
\left(\mathrm{mg}^{-1} \cdot \mathrm{L}^{-1}\right)\end{array}$ & $\begin{array}{c}\text { En. cons. } \\
\left(\mathrm{kWh} \cdot \mathrm{kgO} \mathbf{O}^{-1}\right)\end{array}$ \\
\hline 13,2 & 150,6 & 58 & 7,55 & 1,3 & 360 & 2,38 \\
\hline 8,9 & 76,2 & 83 & 7,55 & 1,2 & 528 & 1,63 \\
\hline 6,1 & 40,8 & 96 & 7,55 & 1,1 & 624 & 1,37 \\
\hline 4,2 & 20,4 & 103 & 7,55 & 1,0 & 678 & 1,26 \\
\hline 3,1 & 11,4 & 114 & 7,55 & 1,0 & 750 & 1,14 \\
\hline 2,2 & 6,0 & 120 & 7,55 & 1,0 & 780 & 1,10 \\
\hline 0 & 0 & 130 & 7,55 & 1,0 & 858 & 1,00 \\
\hline
\end{tabular}

SST: sólidos suspensos totais; TCO: taxa de consumo de oxigênio; $\mathrm{K}_{\mathrm{la}}$ : constante de transferência de oxigênio; $\mathrm{OD}_{\text {ș }}$ : concentração de oxigênio de saturação; $\mathrm{OD}_{c}$ : concentração de oxigênio crítica; $\mathrm{CO}$ : capacidade de oxigenação; En. Cons. energia consumida.

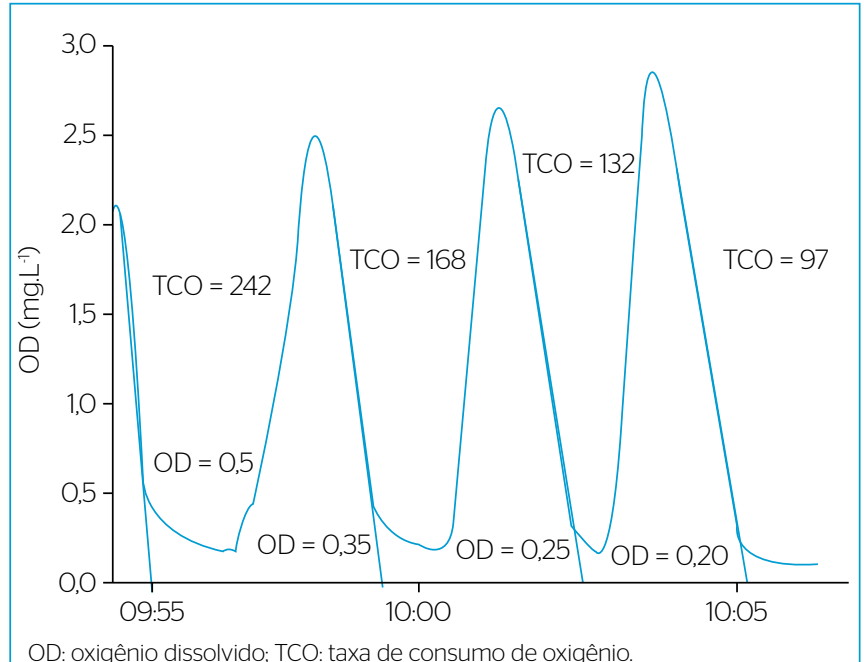

Figura 3 - Concentração de oxigênio em função do tempo em um teste realizado com lodo aplicando aeração intermitente. 
relação empírica foi encontrada a uma temperatura de $25^{\circ} \mathrm{C}$, como expressa a Equação 6.

$\mathrm{OD}_{\mathrm{m}}=\mathrm{TCO} / 500\left(\mathrm{TCO}\right.$ em mg.L $\left.\mathrm{L}^{-1} \mathrm{~h}^{-1}\right)$

É importante salientar que essa é uma relação empírica, portanto com validade restrita às condições experimentais semelhantes às aplicadas durante a investigação. Concluiu-se que a concentração de OD só é limitante quando o seu valor é muito baixo. Os resultados foram obtidos com lodo ativado em suspensão. Se sistemas como MBBR forem usados, os resultados poderão ser muito diferentes (ODEGAARD; GISVOLD; STRICKLAND, 2000). Para estimar a concentração correspondente de oxigênio dissolvido crítico (ODcr) em um reator em escala completa, supôs-se que a estratificação de OD no reator fosse $1 \mathrm{mg} . \mathrm{L}^{-1}$, de modo que a concentração crítica foi calculada como na Equação 6a:

$\mathrm{OD}_{\mathrm{c}}=1+\mathrm{OD}_{\mathrm{m}}=1+\mathrm{TCO} / 500$

Os valores de $\mathrm{OD}_{\text {cr }}$ obtidos para as diferentes concentrações de lodo são apresentados na coluna 5 da Tabela 2. Os dados apresentados na Tabela 2 foram utilizados para estabelecer a influência da concentração de lodo sobre a capacidade de aeração. Uma vez identificados os valores de $\mathrm{K}_{\mathrm{la}}$, $\mathrm{OD}_{\mathrm{s}}$ e $\mathrm{OD}_{\mathrm{cr}}$ (colunas 3, 4 e 5, respectivamente) em função da concentração de lodo, os valores correspondentes da capacidade de aeração foram calculados (coluna 6).

$\mathrm{Na}$ Figura 4, os valores obtidos para a capacidade de aeração são representados graficamente em função da concentração de lodo. Com os dados obtidos para o experimento, gerou-se um gráfico no qual observa-se que há uma tendência de relação linear entre a capacidade de aeração e a concentração de lodo, expressa pela Equação 7:

$\mathrm{CO}_{\mathrm{m}}=858-38^{\star} \mathrm{SST}\left(\mathrm{R}^{2}=0,996\right)$

A partir dessa equação, o valor extrapolado da capacidade de aeração pode ser estimado para um sistema sem lodo $(\mathrm{SST}=0)$ : $\mathrm{CO}_{\text {máx }}=$ $858 \mathrm{mg} \cdot \mathrm{L}^{-1} \cdot \mathrm{h}^{-1}$. Já o consumo comparativo de energia para qualquer concentração poderá ser calculado como na Equação 8:

$\mathrm{E}_{\mathrm{x}} / \mathrm{E}_{\min }=\mathrm{CO}_{\text {max }} / \mathrm{CO}_{\mathrm{m}}=858 /\left(858-38^{\star} \mathrm{SST}\right)$

Em que:

$\mathrm{E}_{\mathrm{x}}=$ consumo de energia para aeração para uma concentração de lodo X;

$\mathrm{E}_{\min }=$ menor consumo para concentração de lodo zero;

$\mathrm{CO}_{\mathrm{x}}=$ capacidade de aeração para concentração de lodo X;

$\mathrm{CO}_{\max }=858 \mathrm{mg} \cdot \mathrm{L}^{-1} \cdot \mathrm{h}^{-1}=$ extrapolação $\mathrm{CO}$ para SST $=0$.
Os valores relativos ao consumo de energia para as diferentes concentrações estão apresentados na coluna 7 da Tabela 2. A Figura 5 mostra os valores experimentais, bem como os valores calculados a partir da Equação 8. É evidente, a partir da coluna 7 da Tabela 2 e da Figura 5 , que o consumo de energia é fortemente afetado pela concentração de lodo: o consumo de energia para uma concentração de $15 \mathrm{~g}$ é aproximadamente 3 vezes mais elevado do que para concentrações mais baixas.

A Tabela 3 mostra uma série de cálculos para avaliar o efeito da concentração de lodo sobre os custos envolvendo o tratamento. Nesse contexto, os custos do tratamento são entendidos como os custos de investimentos anuais gastos com a construção do reator e a energia necessária para a aeração. Considera-se então que outros fatores de custo não são influenciados pela concentração de lodo. O procedimento de cálculo é realizado para uma vazão de esgoto de $1 \mathrm{~m}^{3} \cdot \mathrm{d}^{-1} \mathrm{e}$ pode ser resumido como se segue:

1. Inicialmente, as condições operacionais e a composição do esgoto devem ser conhecidas ou adotadas. Para os cálculos da Tabela 3, ficou estabelecida uma concentração de demanda química de oxigênio (DQO) de $600 \mathrm{mg} \cdot \mathrm{L}^{-1}$, sendo adotadas frações de 10\% para cada um dos seguintes elementos: material orgânico não biodegradável, material solúvel e fração particulada. Supõe-se, ainda, a uma idade de lodo de 12 dias, a uma temperatura de $25^{\circ} \mathrm{C}$. Sob essas condições, a teoria de lodo ativado (VAN HAANDEL \& VAN DER

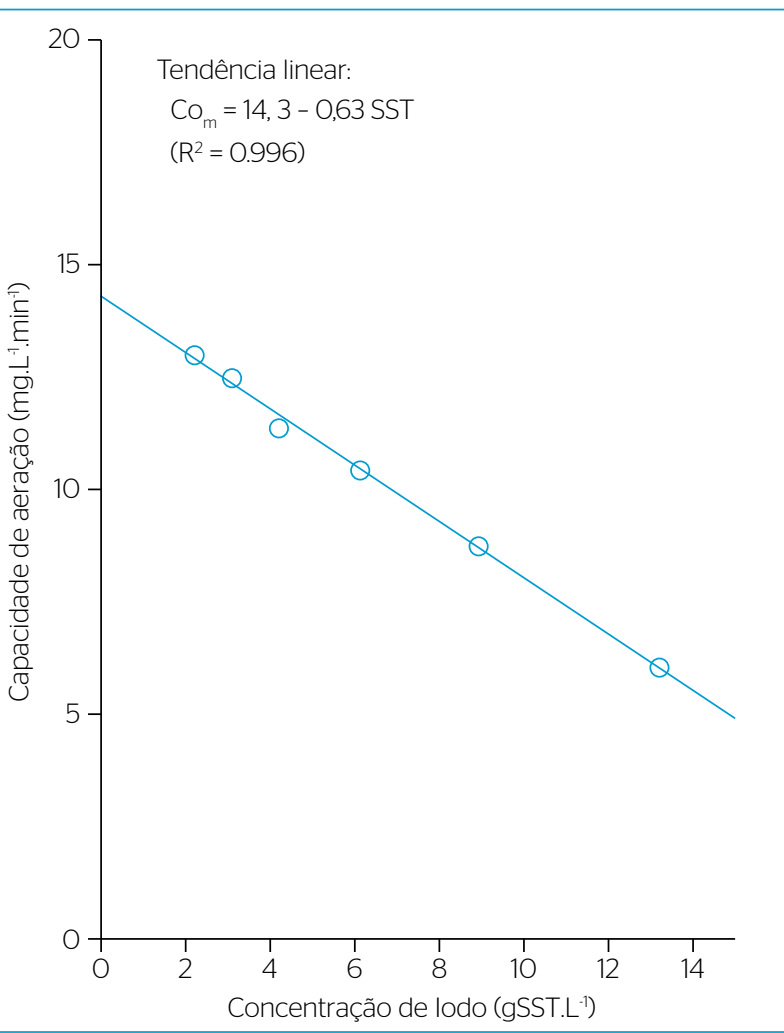

Figura 4 - Diminuição da capacidade de aeração em função da concentração de lodo. (dados da Tabela 2). 
LUBBE, 2012) prevê um consumo de oxigênio de $0,60 \mathrm{kgO}_{2} / \mathrm{kgDQO}$ e massa de lodo de 1,93 kgSST para uma suposta relação sólidos suspensos voláteis (SSV)/SST de 0,75 . No entanto, se a nitrificação

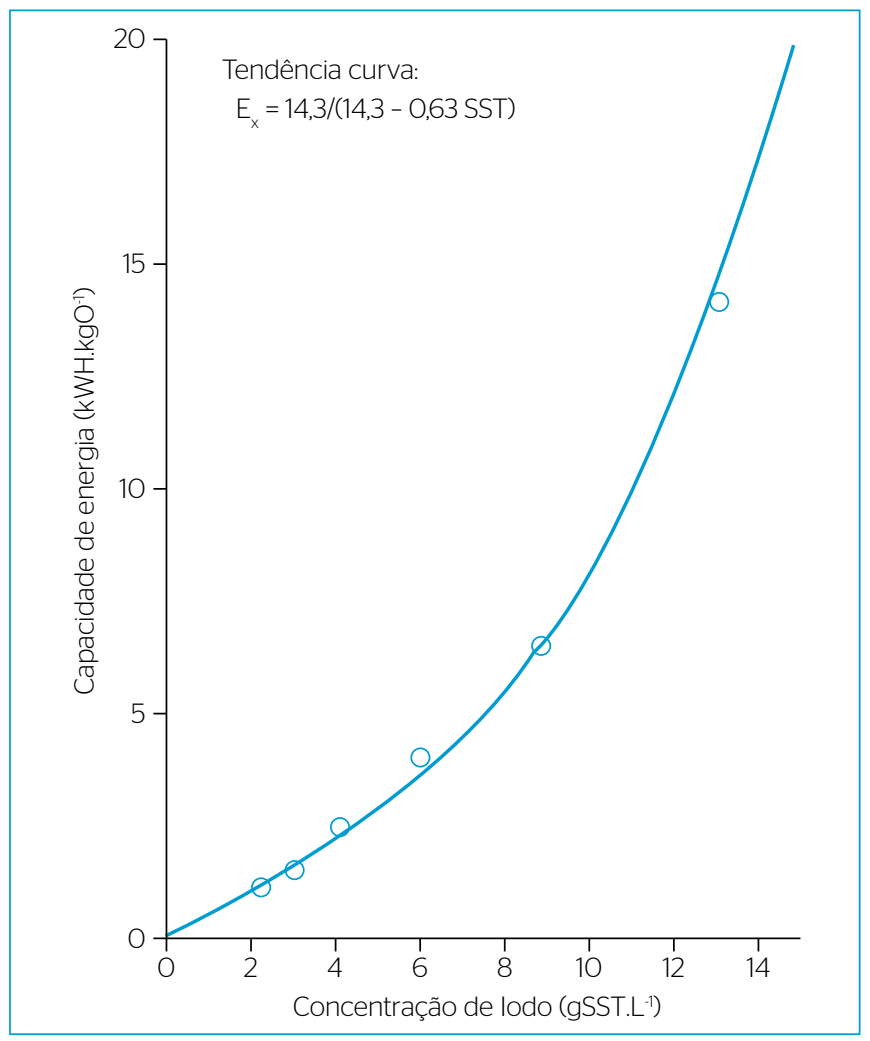

Figura 5 - Consumo relativo de energia para transferir oxigênio em função da concentração de lodo.

Tabela 3 - Procedimento de cálculo para avaliar o custo total da produção e do consumo de energia em função da concentração de lodo.

\begin{tabular}{|c|c|c|c|c|c|c|c|c|}
\hline 1 & 2 & 3 & 4 & 5 & 6 & 7 & 8 & 9 \\
\hline $\begin{array}{l}\text { TDH } \\
\text { (h) }\end{array}$ & $\begin{array}{c}V_{r} \\
\left(m^{3}\right)\end{array}$ & $\begin{array}{l}\text { Const } \\
\text { US\$/ano }\end{array}$ & $\begin{array}{c}X_{t} \\
\left(g . L^{-1}\right)\end{array}$ & $\begin{array}{l}\text { Energia } \\
\text { Wh.gO-1 }\end{array}$ & $\begin{array}{c}\text { Energia } \\
\text { kWh. } \\
\text { ano }^{-1}\end{array}$ & $\begin{array}{c}\text { Aeração } \\
\text { U\$/ano }\end{array}$ & $\begin{array}{c}\text { Total } \\
\text { U\$ano }\end{array}$ & $\begin{array}{c}\text { TCO } \\
\mathrm{mg} \cdot \mathrm{L}^{-1} \cdot \mathrm{h}^{-1}\end{array}$ \\
\hline 12,0 & 0,500 & 50,00 & 3,86 & 1,21 & 237 & 23,68 & 73,68 & 44,9 \\
\hline 11,0 & 0,458 & 45,83 & 4,22 & 1,23 & 241 & 24,13 & 69,96 & 48,9 \\
\hline 10,0 & 0,417 & 41,67 & 4,64 & 1,26 & 247 & 24,69 & 66,36 & 53,8 \\
\hline 9,0 & 0,375 & 37,50 & 5,15 & 1,29 & 254 & 25,42 & 62,92 & 59,8 \\
\hline 8,0 & 0,333 & 33,33 & 5,80 & 1,34 & 264 & 26,39 & 59,72 & 67,3 \\
\hline 7,0 & 0,292 & 29,17 & 6,62 & 1,41 & 277 & 27,74 & 56,91 & 76,9 \\
\hline 6,0 & 0,250 & 25,00 & 7,73 & 1,52 & 298 & 29,79 & 54,79 & 89,7 \\
\hline 5,0 & 0,208 & 20,83 & 9,27 & 1,69 & 332 & 33,22 & 54,06 & 107,7 \\
\hline 4,0 & 0,167 & 16,67 & 11,59 & 2,04 & 402 & 40,16 & 56,83 & 134,6 \\
\hline 3,0 & 0,125 & 12,50 & 15,46 & 3,13 & 616 & 61,59 & 74,09 & 179,4 \\
\hline 5,2 & 0,217 & 21,67 & 8,92 & 1,65 & 324 & 32,36 & 54,03 & 103,5 \\
\hline 11,5 & 0,479 & 47,92 & 4,03 & 1,22 & 239 & 23,89 & 71,81 & 46,8 \\
\hline
\end{tabular}

TDH: tempo de detenção hidráulica; $\mathrm{V}_{\text {: }}$ volume do reator; Const.: custo;

$X_{t}$ : concentração de lodo; TCO: taxa de consumo de oxigênio. fosse incluída (consumo total $=50 \%$ do consumo carbonáceo), o consumo total de oxigênio seria de $0,90 \mathrm{kgO}_{2} / \mathrm{kgDQO}$;

2. Para diferentes valores do tempo de retenção hidráulica (TDH, coluna 1), o volume do reator $\left(\mathrm{V}_{\mathrm{r}}\right.$, coluna 2$)$ é calculado para a vazão adotada de $1 \mathrm{~m}^{3} \cdot \mathrm{d}^{-1}: \mathrm{V}_{\mathrm{r}}=\mathrm{Q}_{\mathrm{i}} / \mathrm{TDH}$;

3. O custo de construção do reator é estimado em 1.000 US\$ por $\mathrm{m}^{3} \mathrm{de}$ reator, e se supõe que os custos anuais sejam equivalentes a $10 \%$ do custo de investimento ao longo de um período de 20 anos, tempo de vida útil do projeto (VAN HAANDEL \& VAN DER LUBBE, 2012). O custo anual está na coluna 3 ;

4. A concentração de lodo é agora calculada na coluna 4, como a razão entre a massa de lodo (1,93 kg.SST) e o volume do reator (coluna 2);

5. Na coluna 5, é calculado o consumo de energia por kg de oxigênio transferido. Para estimar esse parâmetro, o seguinte raciocínio é aplicado:

- Supõe-se que o consumo mínimo de energia $\left(\mathrm{E}_{\text {mim }}\right)$ seja $1 \mathrm{kWh} /$ $\mathrm{kgO}_{2}$ quando a concentração é zero.

- O consumo de energia em qualquer concentração pode ser calculado a partir da Equação 8.

6. Para o consumo de oxigênio estimado de $0,90 \mathrm{kgO}_{2} / \mathrm{KgDQO}$, necessita-se de uma quantidade de oxigênio anual que atenda $1 \mathrm{~m}^{3} \cdot \mathrm{d}^{-1}$ ou $196 \mathrm{kgO}_{2}$. no $^{-1}$. Os valores do consumo de energia são calculados na coluna 6 ;

7. O custo anual de aeração está na coluna 7 , calculado para uma tarifa presumida de $100 \mathrm{US} \$ / \mathrm{MWh}$;

8. O custo total de construção mais aeração está calculado na coluna 8 , resultado da soma das colunas 3 (custo anualizado de investimento) e 7 (custo de aeração).

9. Na coluna 9 está a média da taxa de consumo de oxigênio: a TCO é calculada como a razão entre a transferência de oxigênio por dia $\left(0,9 \mathrm{kgO}_{2} / \mathrm{d}\right)$ e o volume do reator (coluna 2).

Os valores para tempos de retenção curtos são tão elevados que, na prática, pode ser muito difícil conseguir a taxa de transferência desejada, a menos que se aplique um sistema de aeração especial (por exemplo, oxigênio puro). Os valores da TCO na coluna 9 são os valores médios, mas, na realidade, a TCO varia porque a vazão e a concentração de material orgânico variam. Portanto, a TCO máxima que deve ser atendida é maior que o valor calculado na coluna 9.

Vários dos fatores adotados para calcular a relação entre o custo do tratamento e da concentração de lodo podem ser diferentes em aplicações em escala real, mas a tendência de um custo mínimo para uma concentração ótima persiste, independentemente dos valores numéricos.

O custo total (coluna 8 da Tabela 3) mostra que existe um custo mínimo para uma concentração de lodo ótima. Por tentativas e erros, essa concentração é encontrada como sendo 8,92 g.L.-1 para um tempo 
de retenção de 5,2 h (penúltima linha da Tabela 3). O custo mínimo é 54,03 US\$.ano ${ }^{-1}$ para $1 \mathrm{~m}^{3} \cdot \mathrm{d}^{-1}$ de esgoto, ou seja: $0,15 \mathrm{US} \$ . \mathrm{m}^{-3}$. Na prática, provavelmente não é possível operar um sistema de lodo ativado com uma concentração tão alta, a menos que processos como MBR ou MBBR sejam aplicados.

A viabilidade econômica desses processos pode agora ser avaliada com o seguinte exemplo: suponha-se que seja possível operar um sistema de lodo ativado convencional, a uma concentração de lodo de 4 g.L $\mathrm{L}^{-1}$, com um tempo de retenção de 11,5 h, tendo, portanto, um custo de

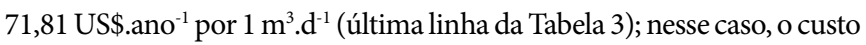
extra comparado com o custo para a concentração ótima $\left(8,92\right.$ g.L $\left.\mathrm{L}^{-1}\right)$ é de 71,81 - 54, $03=17,78$ US\$.ano ${ }^{-1}$ para $1 \mathrm{~m}^{3} . \mathrm{d}^{-1}$. O volume do reator a um TDH $=5,2 \mathrm{~h}$ ou $0,22 \mathrm{~m}^{3} /\left(\mathrm{m}^{3} \cdot \mathrm{d}^{-1}\right)$ é de $0,22 \mathrm{~m}^{3}$ por $\mathrm{m}^{3} \cdot \mathrm{d}^{-1}$. Para o volume de um reator, a diferença de custo é $17,78 / 0,22=82$ US\$/ano ${ }^{-1}$. Assim, para haver viabilidade econômica, o custo de implementação do sistema de aumentar a concentração para 8,92 g.L.-1 deve ser inferior a 82 US\$. $\mathrm{m}^{-3}$.ano ${ }^{-1}$.

Adotando-se novamente que o custo anual seja $10 \%$ do investimento durante 20 anos e sabendo-se que o seu benefício em potencial é de 82 US\$. $\mathrm{m}^{-3}$, para haver viabilidade econômica, o custo não deve ser maior que 820 US\$. $\mathrm{m}^{-3}$ e o estação instalada deve ter uma vida útil de 20 anos. Na prática, o custo com o investimento inicial pode variar bastante, podendo ser maior que o valor estipulado inicialmente, enquanto a vida útil pode ser menor que os 20 anos normalmente estimado para o projeto. Conclui-se que, em geral, a viabilidade econômica dos sistemas MBR e MBBR é questionável: os custos de sua implementação são altos se comparados com os benefícios que se obtêm em termos de redução de custos operacionais. Todavia, é possível que esses sistemas sejam implantados em situações em que não há espaço para a implantação de sistemas convencionais ou se deseja um padrão de qualidade maior.

\section{CONCLUSÕES}

1. A capacidade de aeração de aeradores de bolhas finas em sistemas de lodo ativado depende significativamente da concentração de lodo.

2. A diminuição da capacidade de aeração com crescentes concentrações de lodo é devida, principalmente, a uma diminuição da constante de transferência de oxigênio à medida que a concentração de lodo aumenta.

3. Ainda que possa haver razões para a implementação de sistemas MBR ou MBBR, como a falta de espaço ou a necessidade de produzir efluente de alta qualidade, a aplicabilidade desses sistemas, puramente do ponto de vista econômico, é questionável, uma vez que a soma dos custos de investimento e operacionais necessários é elevada.

4. A concentração de lodo em sistemas aeróbios é limitada pela capacidade de oxigenação do aerador, que precisa transferir o fluxo de oxigênio necessário para atender à demanda do sistema.

\section{REFERÊNCIAS}

CATUNDA, S. Y. C.; DEEP, G. S.; VAN HAANDEL, A. C.; FREIRE, R. C. S. (1996) Método de medição contínua da taxa de respiração em sistemas de lodo ativado. In: XI CONGRESSO BRASILEIRO DE AUTOMÁTICA, 1996, São Paulo. Anais. São Paulo. p. 269-274.

DEZOTTI, M.; JÚNIOR SANT'ANNA, G. L.; BASSIN, J. P. (2O11) Processos biológicos avançados para tratamento de efluentes e técnicas de biologia molecular para o estudo da diversidade microbiana. Rio de Janeiro: Interciência. p. 357.

MEDEIROS, U. T. P.; CAVALCANTI, P. F. F.; VAN HAANDEL, A. C. (2008) Optimization of the DO Concentration in Activated Sludge Systems Treating Petrochemical Effluents, Proceedings. 3 IMEBE, Palma de Mallorca, Spain.

METCALF \& EDDY. (2003) Inc. Wastewater engineering: treatment and reuse. 4. ed. McGraw Hill. p.1819.
ODEGAARD, H.; GISVOLD, B.; STRICKLAND, J. (2002) The influence of carrier size and shape in the moving bed biofilm process. Water Science and Technology, v. 41, n.5, p. 383-392, 2002.

VAN HAANDEL, A. C.; VAN DER LUBBE, J. (2012) Handbook biological waste water treatment: design and optimization of activated sludge systems. 2. ed. Londres - UK: IWA Publishing of Alliance House. p. 816.

VAN HAANDEL, A. C.; CATUNDA, S. Y. C. (2013) Respirometria aplicada ao sistema de lodo ativado: teoria e aplicações. Campina Grande - PB. p. 110. No prelo.

VEOLIA ENVIRONNEMENT. (2009) Technology in MBBR. Disponível em: http://www.veolia.com/mbbr/en/technical details/ htm. Acesso em: 19/11/2012. 